\title{
ELECCIONES AL PARLAMENT 2010: FIN DE CICLO EN CATALUÑA
}

DAVID DELGADO RAMOS. 
SUMARIO

1.- INTRODUCCIÓN. 2.- LA CAMPAÑA ELECTORAL. a) Los candidatos. b) Desarrollo de la campaña y encuestas. 3.- RESULTADOS ELECTORALES. a) Datos generales de las Elecciones. b) Totales. c) Por circunscripciones. d) Evolución del voto en las Elecciones al Parlament (1980-2010). 4.- CONCLUSIONES. 


\title{
ELECCIONES AL PARLAMENT 2010: FIN DE CICLO EN CATALUÑA
}

POR

\author{
DAVID DELGADO RAMOS \\ Doctorando en Derecho Constitucional \\ Universidad Complutense de Madrid
}

\section{1.- INTRODUCCIÓN}

El domingo 28 de noviembre, tras cuatro años de complicada legislatura, Cataluña fue llamada a las urnas para elegir a su nuevo Parlamento. Una legislatura marcada por el Estatut y por la esperada sentencia del Tribunal Constitucional ${ }^{1}$, pero también por la grave crisis económica que, al igual que en el resto de España, estaba afectando gravemente a Cataluña, región antaño considerada el motor económico e industrial del país, pero sometida desde hacía algunos años a un gradual proceso de deslocalización empresarial.

Las elecciones auguraban la victoria de Convergència i Unió que, finalmente estaba a las puertas de volver al Palau de la Generalitat, con su secretario general, Artur Mas, a la cabeza, tras dos elecciones anteriores en las que, aun siendo CiU el partido más votado, no pudo formar Gobierno.

En el «Tripartito» se percibía, tras siete años de convivencia política y de responsabilidad de Gobierno, un «aire de fin de ciclo», de cierto hastío. Los últimos

\footnotetext{
${ }^{1}$ Pese a que el principal recurso de inconstitucionalidad contra el Estatut fue interpuesto por el Partido Popular en julio de 2006, interpusieron también recursos el Defensor del Pueblo y las comunidades autónomas de Aragón, Baleares, Murcia, la Rioja y Valencia. Siete recursos en total.
} 
años se habían caracterizado por la profunda divergencia entre sus integrantes, consecuencia de la falta de unión y consenso, que había producido que en muchas de las principales políticas, el Govern no tuviese una única voz, sino la de los tres partidos que lo conformaban: PSC, ERC e IC-V. Partidos cuyo principal vínculo en común, el progresismo de izquierdas, no alcanzaba a obviar lo mucho que les separaba, fundamentalmente a ERC y al PSC: el independentismo frente al federalismo.

Por ello, y ante la más que previsible derrota, los ánimos estaban encontrados, con escasa voluntad de hacer un balance positivo de los años de gobierno y mucho de querer salvar, a título individual y como partido, sus responsabilidades en los muchos errores cometidos, atribuyendo a los otros partidos su directa responsabilidad en la gestión y resolución de los errores y fracasos.

Por otro lado, el Partido Popular de Cataluña, que, tras años de verse convertido en un partido cuasi «residual», — la cuarta fuerza política en el Parlament en las dos últimas elecciones - y tras el cambio de líder producido en 2008 en un complicado Congreso ${ }^{2}$, se enfrentaba a unas capitales elecciones, de lectura nacional, que deseaban mostrar como el "principio del cambio». Cambio que, continuado en las Elecciones Municipales y Autonómicas de 22 de mayo de 2011, culminarían en la derrota total del PSOE y Zapatero en las Elecciones Generales de 2012.

Las Elecciones, en ese sentido, tenían una innegable lectura nacional, puesto que no sólo se valoraba la gestión de la coalición de gobierno en Cataluña de los últimos siete años, sino que se refrendaban también las políticas económicas para salir de la crisis implementadas por el Gobierno de José Luis Rodríguez Zapatero. Lectura que los dirigentes del PSC y del propio PSOE desmentían con objeto de centrar el debate en Cataluña, pero que resultaba inevitable.

\section{2.- LA CAMPAÑA ELECTORAL}

\section{a) Los candidatos}

En lo que respecta a los candidatos, en el PSC optaba a la reelección a José Montilla ${ }^{3}$, quien anticipó en campaña que estas serían sus últimas elecciones

${ }^{2}$ En el Congreso del Partido Popular de Cataluña de julio de 2008 se elegía al sucesor de Daniel Sirera como Presidente del Partido. Frente a la candidata de consenso, Alicia Sánchez-Camacho, se presentó como alternativa Montserrat Nebrera, diputada en el Parlament. Finalmente, fue elegida líder Alicia Sánchez-Camacho, quien obtuvo el 56,7\% de los votos, frente al 43,28\% de Montserrat Nebrera.

${ }^{3}$ Antes de ser President, fue alcalde de Cornellà de Llobregat (1985-2004), Presidente de la Diputación Provincial de Barcelona (2003-2004) y Ministro de Industria, Turismo y Comercio (20042006). 
como candidato, en un desesperado intento de reducir al mínimo el evidente desgaste que mostraba el PSC en las encuestas.

Convergència i Unió, por su parte, presentaba por tercera vez a Artur $\mathrm{Mas}^{4}$ quien, pese a resultar vencedor en número de escaños en los comicios de 2003 y 2006, no pudo formar Gobierno. Estas elecciones constituían pues su tercera y última oportunidad de recuperar la Generalitat para CiU.

En Esquerra Republicana de Catalunya, tercera fuerza política, era candidato Joan Puigcercós 5 , Presidente de ERC. Puigcercós se enfrentaba al difícil reto de vencer los sondeos, que vaticinaban un descalabro de la formación independentista tras un largo ciclo de victorias de la mano de Josep-Lluís Carod-Rovira ${ }^{6}$, su predecesor y mentor y con quien mantenía una difícil relación.

El Partido Popular de Cataluña tenía en su candidata, Alicia Sánchez-Camacho ${ }^{7}$, un reto hercúleo: vencer la denostada imagen «catalanófoba» que el partido tenía en Cataluña desde el primer tripartito y tratar de mantener sus resultados pese al «viento en contra» que suponía la reciente sentencia del Tribunal Constitucional sobre el Estatut.

Iniciativa per Cataluña, por su parte, renovaba su candidato en la persona de Joan Herrera ${ }^{8}$ tras dos elecciones, las de 2003 y 2006, en las que el candidato fue Joan Saura, Presidente de Iniciativa y conseller en las dos legislaturas del Govern tripartito.

${ }^{4}$ Artur Mas fue consejero de Política Territorial y Obras Públicas (1995-1997), consejero de Economía y Finanzas (1997-2001) y conseller en cap (Consejero en jefe, 2001-2003). Desde el año 2000 es Secretario General de Convergència Democràtica de Catalunya (CDC) y desde el año 2001 Presidente de CiU.

${ }^{5}$ Joan Puigcercós fue Portavoz de ERC en el Congreso de los Diputados (2000-2006) y Secretario General de la formación (2004-2008). En 2006 es nombrado conseller de Gobernación, cargo del que dimite en marzo 2008 tras la derrota electoral en las Elecciones Generales, en la que ERC pasa de 8 a 3 diputados. En el Congreso de ERC de junio de ese año, es elegido Presidente de la formación.

${ }^{6}$ El sorprendente éxito electoral de ERC está asociado a Carod como Presidente (20042008). Candidato a la Presidencia de la Generalitat en 2003, consiguió que ERC pasase de tener 12 a 23 escaños, mientras que en las Elecciones Generales de 2004, pasó de 1 a 8 escaños. En las Elecciones al Parlament de 2006, de nuevo candidato de ERC, desciende a 21 escaños, manteniéndose como tercera fuerza política. En las Elecciones Generales de 2008 su formación acusa el desgaste y desciende de los 8 a los 3 escaños, perdiendo 350.000 votos.

${ }^{7}$ Alicia Sánchez-Camacho, senadora por designación del Parlament desde el año 2008, ha sido diputada en el Congreso (2004-2008) y en el Parlament (1999-2004).

${ }^{8}$ Diputado en el Congreso (2004-2010) y Secretario General de Iniciativa desde el año 2008. 
En Ciutadans, el partido más joven del Parlament y sorpresa de las Elecciones de 2006, en las que, sin apenas recursos, obtuvo casi 90.000 votos y tres escaños, fue de nuevo candidato su joven líder, Albert Rivera9

Otros partidos, sin representación parlamentaria pero con encuestas a su favor, luchan por entrar en el arco parlamentario. Son Plataforma per Catalunya $(\mathrm{PxC})$, Solidaritat Catalana per la Independència (SI) y Reagrupament Independentista (RI).

Plataforma per Catalunya presenta como candidato a su líder, Josep Angla$\mathrm{da}^{10}$, quien busca hacerse un hueco entre quienes han visto la inmigración como un problema para el desarrollo, la estabilidad y el progreso de Cataluña.

Solidaritat Catalana per la Independència, partido fundado poco antes de las Elecciones, tiene como cabeza de cartel a su líder, Joan Laporta, abogado y exitoso expresidente del F.C. Barcelona ${ }^{11}$.

Por último, situado ideológicamente — como Solidaritat - en el independentismo de izquierdas se encuentra Joan Carretero ${ }^{12}$, candidato y líder de Reagrupament Independentista, escisión de Esquerra Republicana de Catalunya.

\section{b) Desarrollo de la campaña y encuestas}

La campaña electoral, pese a que el Fallo de la sentencia sobre el Estatut de Cataluña fue hecho público el 28 de junio ${ }^{13}$, no tuvo como eje central el debate identitario, sino la crisis económica y el balance del gobierno «Tripartito».

\footnotetext{
${ }^{9}$ Abogado y portavoz de Ciutadans en el Parlament (2006-2010).

${ }^{10}$ Concejal en el Ayuntamiento de Vic desde 2003 y Consejero del Consejo Comarcal de Osona.

${ }^{11}$ Bajo el mandato de Joan Laporta (2003-2010), el F.C. Barcelona ganó 4 Ligas, 3 Supercopas de España, 2 Ligas de Campeones, 1 Supercopa de Europa, 1 Copa del Mundial de Clubes y 1 Copa del Rey.

${ }^{12}$ Médico, alcalde de Puigcerdà (Girona) entre 1995 y 2003 y exconseller de Gobernación (2003-2006). En 2007 crea Reagrupament como corriente política dentro de ERC, con objeto de situar el independentismo en el centro del debate político en el partido y dejar de comportarse como un «satélite» del PSC. Candidato por Reagrupament a la Presidencia de ERC en el Congreso de junio de 2008, queda en segundo lugar tras Puigcercòs, obteniendo el 27,56\% de los votos. En abril de 2009 abandonará ERC para liderar Reagrupament como partido, no como corriente de Esquerra.

${ }^{13}$ El 28 de junio fue hecho público el fallo del recurso del Partido Popular interpuesto contra el Estatut de Cataluña, tras cuatro años de deliberaciones y seis borradores de sentencia fallidos, en el que se establecía que «Carecen de eficacia jurídica interpretativa las referencias del Preámbulo del Estatuto de Cataluña a Cataluña como nación y a la realidad nacional de Cataluña». Esto es, lo que los grupos nacionalistas e independentistas consideraban el «alma» del Estatut. Además, y entre
} 
Los partidos políticos presentaron sus lemas y programas. Así, CiU escogió un lema en positivo, "Una Catalunya millor», para presentar sus propuestas, centradas básicamente en la economía, pero evidenciando con su lema la necesidad de una ruptura con la gestión del gobierno Tripartito.

El PSC apeló a los valores de la izquierda con el eslogan «Garantia de progres», con el que hacía un balance de su gobierno en clave progresista para tratar de atraer a todos aquellos votantes que, aún desencantados, simpatizasen con la izquierda.

El Partido Popular, por su parte, centró su campaña en la economía y la crisis, como estaba haciendo el partido a nivel nacional, con un claro mensaje «Solucions per a la crisi», consciente de que el paro y la crisis económica son las mayores y más graves preocupaciones de los catalanes.

Esquerra, en cambio, se presenta a su electorado con el lema «Gent valenta», con el que evidenciaba la debilidad electoral que las encuestas le auguraban, al apelar a la valentía, no su gestión en el Govern, para votar por la formación independentista.

Iniciativa per Catalunya-Verds, apeló a sus principios ecologistas con el lema «Verd. Esperança», con el que pretendía retener a sus tradicionales votantes ecosocialistas e independientes, y conectar con el voto joven, más receptivo a los principios del ecologismo.

Por último, Ciutadans, con su mensaje «Rebélate», el único de los presentados por los partidos que no estaba escrito en catalán, invocó los valores que, sin apenas medios, le dieron tres escaños en 2006: los de un partido joven y moderno alejado de la dinámica habitual de los partidos políticos.

La campaña se desarrolló con la extraña sensación de que «todo estaba escrito», que la victoria de CiU parecía ineluctable. El PSC, que palpaba la derrota, afrontó la campaña a la desesperada, tratando de recuperar el voto indeciso que en 2006 le otorgó la victoria. La alta abstención que pronosticaban las encuestas, que aparentemente beneficiaba al centro-derecha, fue combatida desde continuos llamamientos a la participación, para tratar de movilizar el voto de 2006 y el de las Generales de 2008 que, con su más de un millón seiscientos mil votos, otorgó la victoria a Zapatero. Montilla adelantó que estas elecciones eran las últimas

otros, se declaró inconstitucional el uso del catalán como lengua preferente y la creación de un Consejo de Justicia de Cataluña como órgano de gobierno del poder judicial de Cataluña. La sentencia, que fue votada por bloques de artículos, anuló 14 artículos y fijó la interpretación a dar a otros 23 preceptos y cuatro disposiciones para ser considerados constitucionales. El President Montilla, de inmediato, convocó una manifestación, celebrada el 10 de julio y secundada por la mayoría de los partidos políticos catalanes, con el lema «Som una nació. Nosaltres decidim». 
a las que se presentaba, reclamando una segunda oportunidad a su gestión de los últimos cuatro años.

En el debate televisado en el que participaron los candidatos de todos los partidos, los candidatos en liza centraron el debate en la economía, pese a que el candidato de Esquerra, Puigcercòs, trató de centrar el debate retando al candidato de $\mathrm{CiU}$, Artur Mas, a convocar un referéndum sobre la independencia. Montilla y Mas plantearon sus recetas económicas para salir de la crisis, donde Mas expuso su idea de establecer para Cataluña un concierto económico similar al existente en el País Vasco, mientras que Sánchez-Camacho, por el PP, recordó el estado cercano a la bancarrota de las cuentas de la Generalitat por la gestión del Tripartito. Rivera, por Ciutadans, sorprendió a todos al hablar de la corrupción de $\mathrm{CiU}$ y PSC, en referencia a los dos graves casos que han salpicado a ambos partidos: el caso del Palau de la Música y el caso Pretoria.

Poco después, sin embargo, la intención de CiU y PSC de celebrar un debate sólo entre Mas y Montilla fue anulada en el último momento por la Junta Electoral Central tras la reclamación de los otros partidos, que sostenían que lesionaba la «pluralidad». El debate, no obstante, fue anulado no por un posible perjuicio a la pluralidad, sino porque la solicitud de celebración del debate no se había producido con los cinco días de antelación marcados por la normativa electoral.

Con todo, la campaña tuvo un punto de histrionismo difícilmente justificable, que consiguió no obstante que la ciudadanía catalana se interesase algo más por la campaña y subsiguientemente por las elecciones. Así, el videojuego de la líder del PP catalán, Alicia Sánchez-Camacho, en el que se disparaba a inmigrantes; la participación de una actriz porno como «musa» de la campaña de Solidaritat y sobre todo los videos, como el del PSC, en el que votar a Montilla producía un orgasmo o el de CiU, en el que se decía que España «robaba» a Cataluña, aportaron la nota cómica a una tensa campaña electoral. La mercadotecnia electoral llegó a un punto en el que Montilla aparecía en un cartel como «SuperMontilla», el increíble «hombre normal», para hablar de las ayudas a la emancipación de los jóvenes.

En resumen, una campaña en la que se hizo palpable que, «La fatiga general de tripartito y el desencanto de la izquierda suman a favor del cambio» ${ }^{14}$. La práctica ausencia de reivindicaciones nacionales, salvo por parte de ERC, después de la sentencia del Constitucional sobre el Estatut, propició que el debate se centrase en las que quizá eran las mayores preocupaciones de los catalanes: el desempleo y la crisis económica.

${ }^{14}$ Artículo «El cuento del lobo», de Josep Ramoneda, publicado en el diario El País el 23 de noviembre. 


\section{Encuestas}

\begin{tabular}{|c|c|c|c|c|c|c|c|c|c|c|c|c|c|c|}
\hline & \multicolumn{2}{|c|}{ CiU } & \multicolumn{2}{|c|}{ PSC } & \multicolumn{2}{|c|}{ ERC } & \multicolumn{2}{|c|}{ ICV-EUIA } & \multicolumn{2}{|c|}{ PPC } & \multicolumn{2}{|c|}{ SI } & \multicolumn{2}{|c|}{ C's } \\
\hline & $\%$ & Dip. & $\%$ & Dip. & $\%$ & Dip. & $\%$ & Dip. & $\%$ & Dip. & $\%$ & Dip. & $\%$ & Dip. \\
\hline CIS & 20,6 & & 13,4 & & 5.8 & & 5.4 & & 5.1 & & 0.7 & & 2.4 & \\
\hline $\begin{array}{l}\text { L PERIÓDICC } \\
\text { CATALUNY }\end{array}$ & 39 & $62-63$ & 21,3 & $31-32$ & 7.7 & $11-12$ & 9 & $11-12$ & 10.8 & $14-15$ & 2.7 & $0-2$ & 3.2 & $3-4$ \\
\hline RAC1 & 99,29 & $62-63$ & 20,07 & $30-31$ & 5,62 & 10-11 & 6,68 & $9-10$ & 10.46 & $14-15$ & 2,77 & $2-4$ & 3,17 & 3 \\
\hline$A B C$ & 40 & $60-62$ & 21,7 & 31 & 6,6 & 11 & 10 & $12-13$ & 11,3 & $15-16$ & 1,8 & $0-1$ & 4,6 & 5 \\
\hline LA VANGUARDIA & 39,3 & $63-65$ & 20 & $31-32$ & 7,9 & $10-12$ & 7,5 & $9-10$ & 10,1 & 14 & & $0-1$ & & 4 \\
\hline EL IIUNONO & 40,4 & $61-64$ & 21,8 & $30-32$ & 8,1 & 11 & 8,1 & $9-10$ & 12,7 & $17-19$ & & & 3 & 3 \\
\hline EL PAIS & 39,5 & $64-65$ & 20,4 & 30 & 7 & $8-9$ & 6,9 & 10 & 9,5 & $13-14$ & 2,6 & $1-3$ & 5 & $6-7$ \\
\hline LA RAZON & 39,4 & $60-62$ & 21,8 & $31-32$ & 9,3 & 13-14 & 8,5 & 11-12 & 11,4 & $15-16$ & 1,8 & 0 & 3,2 & $2-3$ \\
\hline PÚBLICO & 39,3 & 62 & 22,22 & 33 & 9,1 & 13 & 8 & 10 & 8,5 & 12 & 2,9 & 1 & 4,1 & 4 \\
\hline
\end{tabular}

RESULTADOS DEFINITIVOS

\begin{tabular}{|ll|ll|ll|ll|ll|ll|ll|}
\hline 38,43 & $\mathbf{6 2}$ & 18,38 & $\mathbf{2 8}$ & $\mathbf{7}$ & $\mathbf{1 0}$ & 7,37 & $\mathbf{1 0}$ & 12,37 & $\mathbf{1 8}$ & 3,29 & $\mathbf{4}$ & 3,39 & 3 \\
\hline
\end{tabular}

Las encuestas reproducidas, publicadas entre el 13 y el 22 de noviembre, vaticinaban sin excepción la derrota de la coalición gobernante, particularmente del PSC, el socio mayoritario, y de ERC, toda vez que Joan Saura, el fuertemente cuestionado conseller de Interior, de Iniciativa per Catalunya-Verds, habia anunciado que no se presentaba como candidato, con lo que IC-V habia mitigado el desplome que también estaba sufriendo. Los medios también coincidian unánimemente en la rotunda victoria de CiU, aunque ninguno auguraba la mayoría absoluta. Por lo que respecta al PP y a Ciutadans, la mayoría de las encuestas le otorgaban un leve aumento, de uno o dos escaños, aunque algunas les otorgaban un descenso. En lo referente a Solidaritat, la tónica general era la de una estimación media de en torno a uno o dos escaños.

Salvo el diario Público, ningún medio de comunicación de los arriba señalados acertó con sus estimaciones sobre $\mathrm{CiU}$, mientras que todos se equivocaron en sus estimaciones sobre el PSC, al otorgarle de media dos o tres escaños más de los finalmente obtenidos. En lo referido al PP, sólo el periódico El Mundo se aproximó finalmente a sus resultados, al otorgarle una horquilla de 17-19 escaños. Los resultados de IC-V fueron anticipados por el diario El País, mientras que a los de ERC se aproximaron $R A C 1$ y el periódico La Vanguardia, que le concedían una estimación de 10-11 y 10-12 escaños, respectivamente. RAC1 también se aproximó a los resultados de Solidaritat al asignarle entre 2 y 4 escaños, mientras que acertó plenamente en sus estimaciones sobre Ciutadans, al igual que El Mundo. 


\section{3.- RESULTADOS ELECTORALES}

Como punto de partida, desde un punto de vista jurídico hay que señalar que Cataluña, en ausencia de legislación electoral propia, se rige por lo establecido en la Ley Orgánica 5/1985, de 19 de junio, del Régimen Electoral General, tal y como se recoge en la Disposición Transitoria Cuarta del derogado Estatuto catalán de $1979^{15}$. El nuevo Estatuto de 2006 mantiene, no obstante, en su Disposición Transitoria Segunda ${ }^{16}$, su vigencia como régimen transitorio.

El Parlament se compone de 135 diputados, elegidos entre las cuatro provincias catalanas con la siguiente división: 85 para Barcelona, 18 para Tarragona, 17 Girona y 15 para Lleida.

a) Datos generales de las Elecciones ${ }^{17}$

\begin{tabular}{c|cc|cc|c|} 
& ELECCIONES 2010 & $\%$ & ELECCIONES 2006 & $\%$ & Var. 06/10 \\
\hline CENSO & 5.363 .688 & & 5.321 .274 & & 42.414 \\
VOTANTES & 3.152 .630 & $58,78 \%$ & 2.982 .108 & $56,04 \%$ & $170.522(2,74 \%)$ \\
VOTOS A CANDIDATURAS & 3.038 .645 & $96,38 \%$ & 2.908 .290 & $97,52 \%$ & $130.355(-1,14 \%)$ \\
ABSTENCIÓN & 2.211 .058 & $41,22 \%$ & 2.339 .166 & $43,96 \%$ & $-128.108(-2,74 \%)$ \\
VOTOS NULOS & 22.354 & $0,71 \%$ & 13.574 & $0,46 \%$ & $8.780(0,25 \%)$ \\
VOTOS EN BLANCO & 91.631 & $2,91 \%$ & 60.244 & $2,02 \%$ & $31.387(0,89 \%)$
\end{tabular}

En lo referente a los datos generales de los comicios, pese al clima de hastío social y desconfianza ciudadana hacia la clase política catalana, que anunciaba una alta abstención, el aumento de la participación constituyó la primera sorpresa de la noche. Participación que se situó en un 58,78\%, incrementándose en un 2,74\% respecto de los comicios de 2006 y que, por inesperada, había obligado a trasladar el derbi Real Madrid-F.C.Barcelona, previsto para el domingo 28 de no-

${ }^{15}$ La necesidad de un régimen electoral propio ha sido una reclamación constante de algunos sectores de la ciudadanía y de algunos partidos políticos. Sin embargo, desde 1980, fecha de las primeras elecciones, ha sido imposible. La distorsión del sistema produce que la provincia de Barcelona, que concentra el $73 \%$ del censo de Cataluña, tenga tan sólo 85 diputados, el $63 \%$ de los 135 en juego. Por el contrario, Tarragona, con el 10,73\% de la población catalana, tiene el 13,33\% de los escaños y Girona, con el $10 \%$ del censo, el 12,59\%. El caso más flagrante es Lleida que, con el 5,62\% de la población de Cataluña tiene el 11,11\% de los escaños en el Parlament.

${ }^{16}$ Disposición Transitoria Segunda del Estatut de Cataluña de 2006: Las disposiciones transitorias tercera, cuarta y sexta de la Ley Orgánica 4/1979, de 18 de diciembre, de Estatuto de Autonomía de Cataluña, mantienen, en lo que corresponda, la vigencia como regulación transitoria».

${ }^{17}$ Datos obtenidos del Departament de Governació i Relacions Institucionals, Generalitat de Cataluña. 
viembre, al lunes 29 , con objeto de fomentar la afluencia de votantes a las urnas. Con todo, a pesar del aumento de la participación, ésta siguió siendo inferior a la media de las elecciones al Parlament desde los primeras elecciones catalanas de 1980, situada en el 60,17\%.

Por otro lado, se produjo un incremento tanto de los votos nulos, del $64,68 \%$, como de los votos en blanco, del $52,10 \%$, incrementos que debieran inducir a una reflexión a la clase política catalana, puesto que constituyen un «termómetro» del grado de desconfianza ciudadana en sus partidos políticos.

\section{b) Totales}

\begin{tabular}{|c|c|c|c|c|c|c|}
\hline & \multicolumn{3}{|c|}{ ELECCIONES 2010} & \multicolumn{3}{|c|}{ ELECCIONES 2006} \\
\hline & votos & ESCAÑOS & $\%$ & votos & ESCAÑOS & $\%$ \\
\hline CONVERGÉNCIA I UNIÓ & 1.202 .830 & 62 & 38,43 & 935.756 & 48 & 31,52 \\
\hline PSC & 575.233 & 28 & 18,38 & 796.173 & 37 & 26,82 \\
\hline PARTIDO POPULAR & 387.066 & 18 & 12,37 & 316.222 & 14 & 10,65 \\
\hline IC-V & 230.824 & 10 & 7 & 282.693 & 12 & 9,52 \\
\hline ERC & 219.173 & 10 & 7 & 416.355 & 21 & 14,03 \\
\hline SOLIDARITAT CATALANA & 102.921 & 4 & 3,29 & & & \\
\hline CIUTADANS & 106.154 & 3 & 3,39 & 89.840 & 3 & 3,03 \\
\hline
\end{tabular}

Los resultados electorales cumplieron en gran medida los pronósticos, y CiU se alzó con el triunfo, con 62 escaños ${ }^{18}$, cercano a la mayoría absoluta, situada en los 68 escaños. El PSC, el gran derrotado, decía adiós al Govern con la peor derrota de toda su historia, 28 escaños, y la pérdida de más de 220.000 votos. ERC, por su parte, sufrió un descalabro electoral sin precedentes, al pasar de 21 a 11 escaños y perder casi 200.000 votos. El PP, el otro vencedor de la noche, constituyó la otra gran sorpresa, al pasar de 14 a 18 escaños, su mejor resultado, y ganar más de 70.000 votos. Iniciativa, el «hermano menor» de Tripartito, aguantó el envite y fue el socio del Govern saliente menos perjudicado por las urnas, al perder sólo algo más de 51.000 votos y 2 escaños y conseguir algo más de 230.000 votos y 10 escaños $^{19}$. Ciutadans mantuvo sus tres escaños, aunque con-

${ }^{18} \mathrm{Al}$ ser CiU una federación, el peso político de Convergència y el de Unió es difícil de dilucidar. A tenor de los resultados y de la lista conjunta que presentaron, se puede considerar que de los 62 diputados obtenidos, 45 corresponden a Convergència Democràtica de Catalunya (CDC) y 17 a Unió Democràtica de Catalunya (UDC).

${ }^{19}$ Iniciativa per Catalunya-Verds, que es una coalición de partidos, a diferencia de CiU, que es una federación, se presenta junto con la rama catalana de Izquierda Unida, Esquerra Unida i Alternativa (EUiA), en todas las elecciones. De los 10 escaños obtenidos, 8 corresponden a Iniciativa y 2 a Esquerra Unida. 
siguió 16.314 votos más. La última sorpresa, Laporta y su partido, Solidaritat, entraron en el Parlament, con cuatro escaños gracias a sus 102.921 votos.

\section{c) Por circunscripciones}

\section{Barcelona}

\begin{tabular}{c|ccc|ccc} 
& \multicolumn{3}{|c}{ ELECCIONES 2010 } & \multicolumn{3}{c}{ ELECCIONES 2006 } \\
& VOTOS & ESCAÑOS & \% & VOTOS & ESCAÑOS & $\%$ \\
\hline CONVERGÉNCIA I UNIÓ & 862.010 & 35 & 36,8 & 664.723 & 27 & 29,89 \\
PSC & 449.549 & 18 & 19,19 & 620.601 & 25 & 27,9 \\
PARTIDO POPULAR & 301.440 & 12 & 12,87 & 248.165 & 10 & 11,16 \\
IC-V & 193.545 & 8 & 8,28 & 230.968 & 9 & 10,38 \\
ERC & 148.973 & 6 & 6,36 & 280.566 & 11 & 12,61 \\
SOLIDARITAT CATALANA & 72.693 & 3 & 3,1 & & & \\
CIUTADANS & 89.990 & 3 & 3,84 & 78.525 & 3 & 3,53
\end{tabular}

Por circunscripciones, la batalla, como siempre, se decidió en Barcelona, la mayor circunscripción por número de escaños en juego, 85, donde CiU obtuvo ocho de los catorce escaños y 197.287 votos de los 267.074 votos más que ha ganado en estas elecciones frente a las de 2006.

El PSC perdió 7 escaños y 171.052 votos, lo que supuso una debacle, particularmente porque reflejaba la derrota de uno de los elementos que constituía su mayor fuerza, el municipalismo, aunque consiguió vencer y mantenerse en algunos de sus tradicionales bastiones del Área Metropolitana de Barcelona, como L'Hospitalet, Cornellà de Llobregat o Santa Coloma de Gramenet. De hecho, en las tres comarcas del conocido como «cinturón rojo» de Barcelona (Barcelonés, Vallès Occidental y Baix Llobregat), feudo tradicional de la izquierda, el PSC fue derrotado por primera vez por CiU. Incluso la propia capital, Barcelona, donde el PSC lleva gobernando ininterrumpidamente desde el inicio de la democracia, por vez primera se vio superado por CiU, que ganó en todos los distritos menos en el de Nou Barris, y que superó al PSC en 130.000 votos.

El Partido Popular, que sumó 301.440 votos y doce escaños en la provincia, aumentó su fuerza en el Área Metropolitana de Barcelona y en la ciudad, ganando 53.000 votos más y dos escaños, — de los cuatro más que obtuvo en total- a causa del desgaste a nivel nacional de Zapatero que, sumado al descrédito del Tripartito y de Montilla por su alejamiento general del tradicional «españolismo» del «cinturón rojo» de Barcelona, poblado de emigrantes de Andalucía, Castilla-La Mancha y Extremadura, llevó a muchos de los que votaron al PSC en anteriores comicios a decantarse por el proyecto del PP, que se man- 
tuvo firme en su defensa de la inconstitucionalidad del Estatut y de la unidad de España.

Esquerra Republicana de Catalunya, por su parte, se dejó cinco de los once escaños que perdió en estas elecciones y más de 131.000 votos en Barcelona, recogidos en gran parte por otros sectores del independentismo, como Solidaritat Catalana per la Independència (SI), que obtuvo tres escaños y 72.693 votos y Reagrupament Independentista, que recogió 23.185 votos. CiU, por su parte, recibió parte de los votos que antes fueron a ERC por su decidida apuesta por «el derecho a decidir», que caló en muchos votantes de la formación independentista.

IC-V perdió en la provincia de Barcelona un escaño y 31.423 votos de los algo más de 51.000 que perdió en toda Cataluña, resistiendo la derrota generalizada de la izquierda. La tradicional fidelidad de su electorado, ecosocialista y comunista, evitó una huida hacia otras posiciones, como ERC o incluso el PSC.

Ciutadans, por último, que concentró en Barcelona el grueso de su electorado (el $88,77 \%$ de sus votos), careciendo de relevancia política en Tarragona, Lleida y Girona, aumentó en un 14,60\% el número de votos en la provincia, pasando de los 78.525 votos de 2006 a los 89.990 de 2010 . El incremento se produjo a costa del PSC, donde en algunos de sus tradicionales «feudos» su electorado optó por votar a Ciutadans como opción «españolista» y de centro-izquierda más adecuada para no votar a su otra opción, el Partido Popular, más alejada ideológicamente de sus postulados.

Tarragona

\begin{tabular}{c|ccc|ccc} 
& \multicolumn{3}{|c|}{ ELECCIONES 2010 } & \multicolumn{3}{c}{ ELECCIONES 2006 } \\
& VOTOS & ESCAÑOS & $\%$ & VOTOS & ESCAÑOS & $\%$ \\
\hline CONVERGÉNCIA I UNIÓ & 121.836 & 9 & 39,32 & 93.277 & 7 & 32,43 \\
PSC & 56.481 & 4 & 18,23 & 74.720 & 5 & 25,98 \\
PARTIDO POPULAR & 41.418 & 3 & 13,37 & 31.644 & 2 & 11 \\
IC-V & 15.747 & 1 & 5,08 & 18.729 & 1 & 6,51 \\
ERC & 26.355 & 1 & 8,51 & 50.686 & 3 & 17,62 \\
SOLIDARITAT CATALANA & 10.581 & 0 & 3,41 & & & \\
CIUTADANS & 8.453 & 0 & 2,73 & 6.970 & 0 & 2,42
\end{tabular}

En Tarragona, la segunda circunscripción catalana en número de escaños, con 18, CiU incrementó sus escaños en dos, hasta los nueve y el PP en uno, hasta los cuatro, mientras que el PSC bajó uno y Esquerra sufrió la mayor pérdida, con dos escaños menos y situarse en uno, perdiendo el $48 \%$ de los votos logrados en las elecciones de 2006. IC-V, con una ligera pérdida de votos consigue retener el único escaño que tenía por la provincia. Ciutadans y Solida- 
ritat no obtuvieron representación, aunque Ciutadans logró en Tarragona su mejor resultado fuera de Barcelona, al conseguir el 8.453 votos, el 2,73\%.

\section{Lleida}

\begin{tabular}{c|ccc|ccc} 
& \multicolumn{3}{|c|}{ ELECCIONES 2010 } & \multicolumn{3}{c}{ ELECCIONES 2006 } \\
& VOTOS & ESCAÑOS & $\%$ & VOTOS & ESCAÑOS & $\%$ \\
\hline CONVERGÉNCIA I UNIÓ & 87.052 & 9 & 46,93 & 72.916 & 7 & 39,99 \\
PSC & 27.461 & 3 & 14,8 & 40.097 & 3 & 21,99 \\
PARTIDO POPULAR & 18.960 & 2 & 10,22 & 16.605 & 1 & 9,11 \\
IC-V & 7.423 & 0 & 4 & 12.018 & 1 & 6,59 \\
ERC & 16.966 & 1 & 9,15 & 32.304 & 3 & 17,72 \\
SOLIDARITAT CATALANA & 5.758 & 0 & 3,1 & & & \\
CIUTADANS & 2.757 & 0 & 1,49 & 1.761 & 0 & 0,97
\end{tabular}

Lleida, la provincia menos poblada de Cataluña aunque, como antes se ha señalado, la más sobrerrepresentada en el Parlament, de los quince escaños en juego CiU logró casi el $50 \%$ de los votos (el 46,93\%) y 9 escaños, desde los siete con los que contaba en 2006, sus mejores resultados en términos porcentuales de las cuatro provincias en juego. El PSC, por su parte, acusó la derrota en números de votos, pasando de poco más de 40.000 a 27.461, aunque manteniendo el número de escaños, tres, y convirtiéndose en la única circunscripción en la que el PSC no retrocede en número de diputados. El Partido Popular, por su parte, obtuvo un diputado más con un levísimo incremento de poco más de 2.000 votos mientras que Iniciativa perdió el único que tenía. Por otro lado, Esquerra Republicana perdió casi el $50 \%$ de sus votos al pasar de 32.304 votos a 16.966 , perdiendo dos de sus tres escaños. Solidaritat y Ciutadans tampoco aquí obtuvieron representación.

\section{Girona}

\begin{tabular}{c|ccc|ccc} 
& \multicolumn{3}{|c}{ ELECCIONES 2010 } & \multicolumn{3}{c}{ ELECCIONES 2006 } \\
& VOTOS & ESCAÑOS & $\%$ & VOTOS & ESCAÑOS & $\%$ \\
\hline CONVERGÉNCIA I UNIÓ & 131.932 & 9 & 45,1 & 104.840 & 7 & 38,19 \\
PSC & 41.742 & 3 & 14,27 & 60.755 & 4 & 22,13 \\
PARTIDO POPULAR & 25.248 & 1 & 8,63 & 19.808 & 1 & 7,22 \\
IC-V & 14.109 & 1 & 4,82 & 20.978 & 1 & 7,64 \\
ERC & 26.879 & 2 & 9,19 & 52.799 & 4 & 19,23 \\
jOLIDARITAT CATALANA & 13.889 & 1 & 4,75 & & & \\
CIUTADANS & 4.954 & 0 & 1,69 & 2.584 & 0 & 0,94
\end{tabular}


En Girona, considerada la provincia más independentista o, si se quiere, la más «catalanista», CiU volvió a ganar con su segundo mejor resultado, al obtener 9 de los 17 diputados y alcanzar el 45,1\% de los votos, hasta 131.932 votos. Su más directo competidor, a muy larga distancia, el PSC, obtuvo 41.742 votos y tres escaños, perdiendo un 31,3\% de los votos de 2006. El PP e IC-V, por su parte, mantuvieron su escaño, con un incremento de más del $27 \%$ de los votos en el caso del PP y una pérdida del 32,7\% de los sufragios en el caso de Iniciativa. Solidaritat obtuvo aquí su único escaño fuera de Barcelona con 13.889 votos, el $4,75 \%$, que arrebató a ERC, que sufrió la pérdida dos escaños y casi el $50 \%$ de los votos, para quedarse en dos escaños, consecuencia también de la erosión que le produjo Reagrupament Independentista que, pese a no lograr ningún diputado, obtuvo en la provincia 9.557 votos, el 3,27\%. Ciutadans tampoco obtuvo en Girona representación.

Evolución del voto en las Elecciones al Parlament (1980-2010)

\begin{tabular}{c|ccccccccc} 
& $\mathbf{1 9 8 0}$ & $\mathbf{1 9 8 4}$ & $\mathbf{1 9 8 8}$ & $\mathbf{1 9 9 2}$ & $\mathbf{1 9 9 5}$ & $\mathbf{1 9 9 9}$ & $\mathbf{2 0 0 3}$ & $\mathbf{2 0 0 6}$ & $\mathbf{2 0 1 0}$ \\
\hline CIU & 27,83 & 46,8 & 45,72 & 46,19 & 40,95 & 37,7 & 30,94 & 31,52 & 38,43 \\
PSC & 22,43 & 30,11 & 29,78 & 27,55 & 24,88 & 37,85 & 31,16 & 26,82 & 18,38 \\
PP & & 7,7 & 5,31 & 5,97 & 13,08 & 9,51 & 11,89 & 10,65 & 12,37 \\
ICV & 18,77 & 5,58 & 7,76 & 6,5 & 9,71 & 2,51 & 7,28 & 9,52 & 7,37 \\
ERC & 8,9 & 4,41 & 4,14 & 7,96 & 9,49 & 8,67 & 16,44 & 14,03 & 7 \\
C'S & & & & & & & & 3,03 & 3,39 \\
SI & & & & & & & & & 3,29
\end{tabular}

La evolución del voto en Cataluña desde las primeras Elecciones de 1980 revela la preeminencia de Convergència i Unió como principal fuerza política en Cataluña, obteniendo el mayor número de votos en siete de las nueve elecciones al Parlament que se han celebrado, seis seguidas con Jordi Pujol como cabeza de cartel y presidente de la Generalitat.

El PSC, por su parte, ha resultado vencedor en sólo dos comicios, los de 1999 y 2003, aunque en estos últimos fue superado en escaños por CiU. Sin embargo, el PSC se consolidó como alternativa real a Convergència a medida que apuntalaba sus bastión político por excelencia: la fortaleza de su poder municipal, en el que sin cesar reeditaban mayorías absolutas de la mano de sus «capitanes», los alcaldes de los municipios del área metropolitana de Barcelona y sus comarcas, que conseguían, por su empatía y conexión con el ciu- 
dadano medio de esas poblaciones, obrero y emigrante, sucesivas victorias. Victorias que no eran secundadas a nivel autonómico por la percepción generalizada existente de que el PSC no tenía «fuelle» suficiente para alcanzar la Generalitat.

El Partido Popular, que en los años ochenta no pasó de ser un partido residual, consecuencia de su identificación con el franquismo que tenía la ciudadanía catalana, dio un salto cualitativo hacia la mitad de los noventa, por la pujanza del centro-derecha y el brutal desgaste que asoló al PSOE en los estertores del «felipismo». Así, primero con Vidal-Quadras y después con Piqué, renovaron el «alma» del PP para adaptarlo al seny catalán, en un periodo de tiempo, hasta la pérdida del Govern de CiU en 2003, de fuerte ligazón política.

Esquerra Republicana de Catalunya, en general ha sostenido una progresión electoral desde las elecciones de 1988, con leves altibajos como los de 1999 y 2006, además de la derrota sufrida en esta elecciones de 2010. Saltos cualitativos que se produjeron tras bruscos cambios en el liderazgo del partido, primero de Joan Hortalà por Àngel Colom (1989) y después de Colom por Josep-Lluís Carod-Rovira (1996), que permitieron a la formación renovarse e integrar en su seno al radicalismo juvenil independentista, particularmente tras la disolución del grupo terrorista catalán Terra Lliure en 1995.

Iniciativa per Cataluña-Verds, en la actualidad en coalición con Esquerra Unida i Alternativa (EUiA), ha tenido una historia política bastante tortuosa, fruto de la amalgama de partidos que aglutinaba, entre los que se encontraban el PSUC y el PCC. Sus constantes diferencias con EUiA, que llevaron a la disolución de la coalición en 1998, para recomponerla en 2003. Su evolución, pareja a la crisis de la izquierda, tuvo su punto de inflexión en 1as elecciones de 1999, poco después de la ruptura con EUiA, en la que sólo obtuvo el 2,51\% de los votos y 3 escaños. Desde entonces, hasta la derrota de 2010, ha tenido una progresión constante, hasta llegar a su culmen en 2006, año en que lograron el $9,52 \%$ de los votos y 12 escaños.

\section{4.- CONCLUSIONES}

Las Elecciones al Parlament de 2010 han supuesto el fin de un ciclo político marcado por los gobiernos «Tripartitos» de PSC-ERC-ICV, primero bajo la presidencia de Pasqual Maragall (2003-2006) y después con José Montilla (2006-2010). Gobiernos que, por su inexperiencia en las tareas de gestión y su falta de consenso político, difundían una permanente sensación de inestabilidad, 
inseguridad y desconcierto. El resultado, dos ganadores claros (CiU y PP) y dos claros perdedores (PSC y ERC).

La rotunda victoria de la federación de CiU, con 62 escaños, a tan sólo 6 de la mayoría absoluta y con un incremento de 14 escaños respecto de las Elecciones de 2006, augura un gobierno estable en la Generalitat, con acuerdos puntuales con el Partido Popular y Esquerra Republicana de Catalunya, toda vez que su victoria ha sido total, al ganar en las cuatro capitales de provincia, las cuarenta y una comarcas y en 921 de los 947 municipios catalanes.

En ese sentido, los resultados electorales obtenidos por el Partido Popular le otorgan una extraordinaria posición de fuerza y una importancia incuestionable. Su recobrada posición como tercera fuerza política, tras ser, durante las últimas dos legislaturas, la cuarta, le permite situarse cómodamente en el espacio político. Espacio que sus oponentes, $-\mathrm{y}$ hasta los propios dirigentes del Partidonunca pensaron que alcanzaría, toda vez que salvo alguna encuesta, la percepción general estimaba que el PP subiría, a lo sumo, dos escaños, fundamentalmente por el descrédito de Zapatero y del PSOE, y también a causa de la abstención prevista.

Las previsiones fallaron, lo que demostró que el Partido Popular, en Cataluña, tiene un «oscilante» pero real «voto oculto», que en algunos casos, como el presente, sirve para demostrar que su importancia es más real que aparente, como evidencia la fluctuación del voto entre las elecciones autonómicas y generales $^{20}$.

En cualquier caso, que el Partido Popular de Cataluña, tras un brutal ostracismo político al que se ha visto sometido en esta segunda legislatura del Tripartito, consecuencia de la desfavorable imagen del partido en Cataluña y del recurso de inconstitucionalidad que interpuso contra el Estatut, y a pesar de la sentencia del Tribunal Constitucional, haya obtenido, con mayor participación, los mejores resultados en número de escaños de su historia ${ }^{21}$, pasando de 14 a 18 , tiene un

${ }^{20}$ En las Elecciones al Parlament de 1995, el PP obtuvo 421.752 votos. En las Generales de 1996, 698.400. En las Elecciones al Parlament de 1999, recabó 297.265 votos, frente a 768.318 de las Generales de 2000. En las Elecciones al Parlament de 2003 logró 393.499 votos, mientras que en las Generales de 2004 alcanzó los 626.107 votos. Por último, en las Elecciones al Parlament 2006 consiguió 316.222 votos, por 610.473 votos obtenidos en las Generales de 2008. CiU es la causante de esta oscilación, produciéndose una transferencia de votos entre CiU y PP en función del tipo de elección a la que concurran, por la parcial afinidad del electorado de ambos partidos. Así, prefieren votar más al PP para unas elecciones generales, por ser un partido nacional, frente a unas elecciones al Parlament, donde optan por dar preferencia a CiU.

${ }^{21}$ En cuanto a número de votos, son los terceros mejores resultados del PP de Cataluña. Los mejores resultados en cuanto a número de votos los obtuvo con Aleix Vidal-Quadras como cabeza de car- 
significación notable y además, doble. Primero, porque revela que el PP no es un partido residual en Cataluña. Y segundo, porque muestra la desconfianza de una parte del electorado tradicional del PSC, que ha votado al PP ante la supuesta «traición» de Montilla a su electorado más profundamente españolista. Paradójicamente, aquella parte de su electorado de donde el propio Montilla procede, el llamado «cinturón rojo» de Barcelona, electorado obrero e inmigrante de Andalucía, Extremadura y Castilla-La Mancha. De los algo más de 70.000 votos que ha obtenido el PP con respecto a las Elecciones de 2006, 53.000 provienen de la provincia de Barcelona, consecuencia no sólo debida a que Barcelona es la mayor circunscripción con diferencia, sino al éxodo de votantes desencantados e independientes que habían votado al PSC con anterioridad.

La derrota del PSC, que pasa de 37 a 28 escaños, y la pérdida del Govern, anticipa una larga «travesía en el desierto» para los socialistas catalanes, toda vez que su gestión al frente de la Generalitat ha quedado desacreditada, fundamentalmente por culpa de sus socios de Gobierno y el desgaste de más de seis años de Gobierno del PSOE a nivel nacional, con una gravísima crisis económica que se ha llevado por delante, además de a dos millones de desempleados, -hasta llegar a los 4,7 millones, el 20,33\% ${ }^{22}$ de la población — gran parte de los «dogmas» hasta ahora intocables de socialismo, como la reforma laboral, la retribución de los empleados públicos y la reforma de las pensiones.

Sin embargo, la crisis más fuerte a la que se enfrenta el socialismo catalán y que se recrudecerá en la oposición, es la pugna subyacente, irresoluble en parte desde los tiempos al frente del partido de Raimon Obiols ${ }^{23}$, entre las dos espíritus o almas ${ }^{24}$ que conforman el PSC: el alma españolista y la catalanista. Conflicto que se intensificará, ante la necesidad o la evidencia de una catarsis que sea

tel en las Elecciones de 1995, obteniendo 421.752 votos, el 13,21\%, pero 17 escaños. Los segundos los alcanzó con Josep Piqué como candidato en las Elecciones de 2003, logrando 393.499 votos, el $11,89 \%$, y 15 escaños. Alicia Sánchez-Camacho ha obtenido 387.066 votos, el 12,37\%, y 18 escaños. Un escaño más con 34.686 votos menos que Vidal-Quadras y tres escaños más con 6.433 votos menos que con Piqué, por el sistema electoral catalán. Sin embargo, las posibilidades de éxito del PP y sus dificultades de cara a las Elecciones de 2010 eran mucho mayores que en 1995 y 2003.

${ }^{22}$ Datos de la Encuesta de Población Activa (EPA), correspondientes al cuarto trimestre de 2010.

${ }^{23}$ Raimon Obiols, actualmente eurodiputado, fue primer secretario del PSC entre 1983 y 1996. Candidato a la Presidencia de la Generalitat en las elecciones de 1984, 1988 y 1992, pertenecía al ala más catalanista del PSC.

${ }^{24}$ Este aspecto lo ha sintetizado brillantemente Miquel Porta Perales en un artículo titulado «Las dos almas del PSC» publicado en el diario ABC el pasado día 17 de diciembre. En él, señala que «Desde su fundación —el 16 de julio de 1978, fruto de la unificación del Partit Socialista de Catalunya (Congrés), el Partit Socialista de Catalunya (Reagrupament) y la Federación Catalana del PSOE-, el Par- 
capaz de devolver a los socialistas catalanes al Poder e ilusionar a un electorado que en los últimos quince años, le permitió gobernar, sólo o en coalición, en la Generalitat, las cuatro capitales de provincia de Cataluña y presidir todas las Diputaciones Provinciales.

Por ello, muy probablemente, en el Congreso que celebrarán en otoño de 2011, los socialistas catalanes deberán afrontar una refundación complicada, en sintonía con la complicada situación que vive Cataluña. Refundación que no se solucionaría con la elección de una nueva dirección proveniente del ala «catalanista», puesto que la responsabilidad de la debacle electoral no es sólo de los «españolistas o capitanes» que dirigían el partido desde el Congreso de Sitges, con José Montilla a la cabeza, sino también de todos aquellos que, procedentes o integrantes del sector «catalanista», se plegaron al ideario o a las políticas identitarias de los nacionalistas catalanes de CiU y ERC. La catarsis es pues doble, con objeto de evitar que el PSC, en vez de ser percibido como un partido plural, pero sobre todo, transversal, como lo fue siempre, sea percibido como un partido «bipolar» que, como el dios romano Jano, posee dos caras antagónicas e irreconciliables, una que mira a España y otra, a Cataluña.

El otro gran derrotado de las Elecciones, Esquerra Republicana de Catalunya, tiene ante sí un futuro sombrío. Durante las dos legislaturas de Govern tripartito ha podido mostrar a la ciudadanía que la aupó al poder su forma de gobernar, pero sus numerosos errores han condenado, en gran parte, a un Govern preso de sus «demonios de partido», incapaz de separar la acción política de Gobierno de la dinámica interna de los partidos. Confundieron pues Generalitat y

tit dels Socialistes de Catalunya ha vivido una serie de querellas internas entre las Ilamadas almas catalanista y españolista. Ya en su origen apareció el conflicto entre la tradición socialista catalana y la española. La primera —anticentralista y antiestatista - bebía en las fuentes del catalanismo, el anarcosindicalismo, el cooperativismo, la autogestión y el cristianismo social. La segunda — centralista y estatista-procedía del obrerismo clásico made in PSOE». Se impuso, en el Congreso de 1980, el sector «catalanista», que durante más de una década controló el partido. Por ello, y hasta la elección como primer secretario de José Montilla en el año 2000, todos los líderes del partido procedían del «ala catalanista»: Joan Reventós (1978-1983), Raimon Obiols (1983-1996) y Narcìs Serra (1996-2000). Aunque desde el VII Congreso del Partido, celebrado en Sitges en 1994, el núcleo dirigente pertenecía al «ala españolista», —los llamados «capitanes»— con José Montilla (entonces, alcalde de Cornellà de Llobregat), José Zaragoza (actual secretario de Organización), Miquel Iceta (portavoz del PSC) y Celestino Corbacho al frente (alcalde de L'Hospitalet entre 1994 y 2008 y Presidente de la Diputación Provincial de Barcelona entre 2004 y 2008), en 2003 fue candidato Pasqual Maragall, exalcalde de Barcelona (1982-1997) y Presidente del PSC (2000-2007), además de candidato a la Generalitat en 1999, que procedía del «ala catalanista». Finalmente, en el Congreso del año 2000, toman el control total los «españolistas», lo que no ha sido óbice para que, paradójicamente, el president Montilla haya hecho bandera del «catalanismo» más nacionalista. 
Partido. Además, la batalla por el control de ERC entre Carod y Puigcercòs tras las Elecciones Generales de 2008 se trasladó a la ciudadanía, que observaba atónita el fraccionamiento interno del partido.

En ese sentido, la dimisión de Puigcercòs como Presidente de ERC, y la convocatoria de un nuevo Congreso del partido para otoño, auguran un periodo de relativa paz, antesala de un nuevo liderazgo en la formación independentista. Un liderazgo «tranquilo», alejado de la crispación y el histrionismo de los años de Carod y Puigcercòs, y que podría cobrar forma en la persona de Joan Ridao, Secretario General de Esquerra y bien valorado por las bases. Un cambio que, paradójicamente, tendría que hacer reconducir el partido hacia la senda que marcó Carod, quien consiguió en 2003 y 2006 un resultado espectacular abriendo el partido a la izquierda, con un discurso más izquierdista y relegando al independentismo a un segundo lugar.

Iniciativa per Catalunya, por su parte, ha conseguido, sorprendentemente, mitigar la debacle electoral que, hace apenas unos meses, pronosticaban las encuestas. El cambio de líder, personificado en la carismática figura de Joan Herrera, hábil orador, les ha permitido superar con «dignidad» el desafío electoral, aunque hayan perdido el Govern. Ciertamente, varios meses atrás casi ningún dirigente hubiese apostado por estos resultados tras la nefasta y desacreditada gestión de Saura al frente de la Conselleria de Interior. Sin embargo, Iniciativa ha conseguido 230.824 votos y 10 escaños, por lo que sólo ha perdido 51.869 votos y dos escaños, convirtiéndose en la cuarta fuerza política, por delante de ERC. Aparte del cambio de líder, otra de las claves de su moderada derrota hay que atribuirla, no obstante, al tradicional voto «fiel» de la formación ecosocialista. El reto de Iniciativa, de la mano de Herrera, será lograr encontrar su lugar en la oposición, potenciando su vertiente «verde», toda vez que su perfil progresista se solapa con ERC y, fundamentalmente, con el PSC.

Por otro lado, un nuevo actor entra en el tablero político catalán: Joan Laporta y su partido, Solidaritat Catalana per la Independència (SI). Un partido hecho a la medida de su líder quien, probablemente catalizador del descontento de un sector del independentismo catalán con Esquerra y del espíritu beligerante que inundaba algunos sectores de la sociedad catalana tras la sentencia del Tribunal Constitucional, obtiene 4 escaños (tres de ellos en Barcelona y el cuarto en Girona) y 102.921 votos. Laporta, cobijado bajo el paraguas de su exitosa Presidencia del Fútbol Club Barcelona, ha trasladado a la ciudadanía la imagen de hombre triunfador, capaz de resolver todos los problemas de Cataluña. Pese a su retórica agresiva y sus constantes alusiones a la independencia de Cataluña como solución de todos los problemas, su presencia, lejos de impulsar el independentismo, lo fracciona aún más, como ya se ha podido observar en las elecciones, en 
las que dos partidos más a parte de Solidaritat, batallaban por idéntico electorado: ERC y Reagrupament. Si en 2006 ERC obtuvo, en solitario, 416.355 votos y 21 escaños, en estas elecciones, la suma de ERC, Solidaritat y Reagrupament consiguió 361.928 votos y 14 escaños (10 de ERC y 4 de Solidaritat). En términos absolutos, el independentismo catalán ha perdido sólo 54.427 votos, un $13,07 \%$, que han sido recogidos fundamentalmente por CiU. Su derrota ha sido debida, por tanto, a su división interna, no su ausencia de fuerza.

Muy probablemente, Laporta, novel en el proceloso mundo de la política, pero tremendamente ambicioso, tratará de fagocitar el independentismo catalán con objeto de unirlo bajo su liderazgo, consolidándolo, para definir la agenda política para la consecución de la independencia de Cataluña, único objetivo de Laporta. Solidaritat, en manos de Laporta, no es más que el instrumento necesario para unir a todo el independentismo catalán, que tiene otro ritmo, particularmente Esquerra.

No hay que olvidar que Laporta, antes de fundar Solidaritat, coqueteaba con CiU y ERC y que, cuando descartó ambas opciones, se daba por segura su incorporación a Reagrupament. Discrepancias en torno al liderazgo y la configuración de las listas electorales condujeron finalmente a la ruptura entre Carretero y Laporta, quien decidió buscar otro camino, fundando por ello Solidaritat, junto a Alfons López Tena ${ }^{25}$ y Uriel Bertran. La elección de estos compañeros de viaje no es baladí, puesto que López Tena, dirigente de CDC, pertenecía al sector «independentista» de $\mathrm{CiU}$, mientras que Bertran, exdiputado en el Parlament con ERC y líder de la corriente «Esquerra Independentista», había optado a la Presidencia de ERC en el Congreso de 2008, quedando su candidatura en el último lugar. Pretendía aglutinar pues en su partido todo el «espectro» político independentista, de izquierda a derecha, con objeto de crear un único bloque independentista, liderado por él.

Ciutadans, la sorpresa de las Elecciones de 2006, ha conseguido mantenerse a flote tras la tumultuosa crisis interna ${ }^{26}$ que sufrió durante la mayor

\footnotetext{
${ }^{25}$ Notario y exvocal del Consejo General del Poder Judicial (2001-2008).

${ }^{26}$ Ciutadans se ha visto sometido a una fuerte crisis interna en esta legislatura, entre acusaciones de personalismo a Albert Rivera y falta de democracia interna, lo que ha propiciado que los otros dos diputados de Ciutadans en el Parlament, Antonio Robles y José Domingo, se diesen de baja en el partido. Robles, quien fue secretario general hasta 2007, renunció al acta de diputado en septiembre de 2009, para pasar en noviembre a UPyD como portavoz de la formación en Cataluña y su cabeza de lista para las Elecciones de 2010, donde ha obtenido 5.418 votos, el 0,17\%. José Domingo se mantuvo como diputado no adscrito, en el Grupo Mixto, hasta el fin de la legislatura. Otro motivo de fuerte controversia tuvo lugar con ocasión de las Elecciones al Parlamento Europeo de 2009, donde Ciutadans concurrió en coalición con Libertas, formación euroescéptica del millonario irlandés Declan Ganley que encabezaba en España Miguel Durán, y asociada en el res-
} 
parte de la legislatura, logrando no sólo mantener los resultados de 2006, sino incrementarlos en 16.314 votos, un 18,16\% más que en 2006, manteniendo, no obstante, el número de escaños, tres, aunque rozando el cuarto. El electorado de Ciutadans, alineado con posiciones anti-nacionalistas y constitucionalistas, pero situado en el espectro de independientes y de centro-izquierda, ha visto en la formación la rebeldía de quien no se sitúa en los convencionalismos de la política, por lo que han revalidado su confianza en la formación.

Como último apunte señalar que, en lo que respecta a los resultados obtenidos por los otros dos partidos que a priori partían, - a tenor de las encuestas - con opciones de entrar en el Parlament, Plataforma per Catalunya y Reagrupament, sus resultados, aun no habiéndoles permitido la obtención de representación parlamentaria, no les hacen actores a desdeñar. La fuerza de su electorado, 75.134 votos en el caso de Plataforma per Catalunya y 39.834 votos en el caso de Reagrupament, les sitúa en una complicada situación política para sobrevivir: o se unen a partidos semejantes para potenciar y amplificar su influencia o bien se verán abocados a la desaparición, sobreviviendo como partidos muy residuales, mientras su electorado se diluye en $\mathrm{CiU}$ y PP, - en el caso de Plataforma per Catalunya - y ERC y Solidaritat, —en el caso de Reagrupament-.

La Elecciones al Parlament de Catalunya 2010 han supuesto, en definitiva, el fin del ciclo político del «Tripartito». Un ciclo marcado por el Estatut y su esperada Sentencia por parte del Tribunal Constitucional que, sin embargo, ha oscurecido la gestión política de tres partidos que, tras el Pacto del Tinell en 2003, accedieron, sin experiencia previa en el gobierno de la Generalitat, a conformar un Govern que dejase atrás veintitrés años de ciclo político de CiU bajo el mando de Jordi Pujol. El balance, en general, no ha resultado positivo para Cataluña ni para sus ciudadanos, puesto que se ha producido un fortalecimiento de determinadas cuestiones identitarias ajenas a los reclamos e intereses de la ciudadanía catalana en general. Cataluña no pidió un nuevo Estatut ${ }^{27}$ ni más autogobierno, sino un Govern de marcado contenido social, que llevase a cabo un giro

to de Europa a partidos de la extrema derecha, obteniendo en toda España 22.903 votos, el $0,14 \%$. Esta coalición, contraria según algunos militantes a los principios fundacionales de Ciutadans, produjo que se diesen de baja Antonio Robles y José Domingo.

${ }^{27}$ El Estatut, una vez aprobado por las Cortes el 10 de mayo de 2006, fue sometido a referéndum el 18 de junio. La participación fue del 48,85\% y fue ratificado con el 73,24\% de los votos a favor y el 20,57\% en contra. Es decir, el Estatut fue apoyado por un tercio de los ciudadanos catalanes con derecho a voto, lo que demuestra el escaso interés que suscitó el nuevo texto estatutario. 
a políticas sociales de izquierda, que reforzasen la cohesión social y corrigiesen las desigualdades.

Cataluña no reclamaba una potenciación de la inmersión lingüística ni la amplificación del «supuesto» conflicto con España, que no existía como tal, tan sólo en el imaginario político de algún partido. Simplemente deseaba un reconocimiento a su identidad y mejoras fiscales para consolidar su autogobierno dentro del marco estatutario del Estatut de Sau de 1979.

Cataluña, en suma, votó el 28 de noviembre pasado a favor de la estabilidad política, de la «tranquilidad», y para ello optó por el partido que se la otorgó durante veintitrés años. El partido que, jugando con una calculada ambigüedad entre el soberanismo (Convergència) y el máximo autogobierno posible dentro de España (Unió), es la viva representación del célebre seny catalán, de su sentido común, que es lo que, tras siete años de gobierno del «Tripartito», necesitaba Cataluña 
